\title{
Probing the Wave Function and Dynamics of the Quintet Multiexciton State with Coherent Control in a Singlet Fission Material
}

\author{
S. L. Bayliss $\odot,{ }^{1, \hbar,}$ L. R. Weiss $\odot,{ }^{2, \hbar,}$ F. Kraffert $\odot,{ }^{1}$ D. B. Granger, ${ }^{3}$ J. E. Anthony, ${ }^{3}$ J. Behrends $\odot,{ }^{1, *}$ and R. Bittl $\odot^{1, \dagger}$ \\ ${ }^{1}$ Berlin Joint EPR Lab, Fachbereich Physik, Freie Universität Berlin, D-14195 Berlin, Germany \\ ${ }^{2}$ Cavendish Laboratory, J. J. Thomson Avenue, University of Cambridge, \\ Cambridge CB3 OHE, United Kingdom \\ ${ }^{3}$ Department of Chemistry, University of Kentucky, Lexington, Kentucky 40506-0055, USA
}

(Received 13 January 2020; revised manuscript received 16 April 2020; accepted 30 April 2020; published 29 June 2020)

\begin{abstract}
High-spin states play a key role in chemical reactions found in nature. In artificial molecular systems, singlet fission produces a correlated triplet-pair state, a spin-bearing excited state that can be harnessed for more efficient solar-energy conversion and photocatalysis. In particular, triplet-pair states with overall quintet character (total spin $S=2$ ) have been discovered, but many of the fundamental properties of these biexciton states remain unexplored. The net spin of these pair states makes spin-sensitive probes attractive for their characterization. Combined with their surprisingly long spin coherence (of order microseconds), this opens up techniques relying on coherent spin control. Here we apply coherent manipulation of triplet-pair states to (i) isolate their spectral signatures from coexisting free triplets and (ii) selectively couple quintet and triplet states to specific nuclear spins. Using this approach, we separate quintet and triplet transitions and extract the relaxation dynamics and hyperfine couplings of the fission-borne spin states. Our results highlight the distinct properties of correlated and free triplet excitons and demonstrate optically induced nuclear spin polarization by singlet fission.

DOI: $10.1103 /$ PhysRevX.10.021070

Subject Areas: Chemical Physics, Materials Science, Semiconductor Physics
\end{abstract}

\section{INTRODUCTION}

Interacting triplet-pair states are key intermediates in photocatalysis [1], photon up-conversion [2], recombination in light-emitting diodes [3-5], as well as the process of singlet fission [6,7] - the production of two spin-1 triplet excitons from an initial spin-0 singlet exciton, a process which has received great interest for its potential in photovoltaics [8-11]. Recently, it has been demonstrated that the biexciton generated by the fission process can evolve in time to form a long-lived pair state with total spin $S=2$ : a spin-quintet state [12-19]. This quintet state is intriguing for several reasons. First, while biexciton states typically undergo rapid recombination [20], quintets in organic materials are theoretically spin protected from recombination losses, due to triplettriplet annihilation to the single-molecule quintet state being

*j.behrends@fu-berlin.de

robert.bittl@fu-berlin.de

${ }^{*}$ Present address: Pritzker School of Molecular Engineering, University of Chicago, Chicago, Illinois 60637, USA.

${ }^{\S}$ These authors contributed equally to this work.

Published by the American Physical Society under the terms of the Creative Commons Attribution 4.0 International license. Further distribution of this work must maintain attribution to the author(s) and the published article's title, journal citation, and DOI. energetically inaccessible [21,22]. Second, as a long-lived, high-spin state there is potential to use the coupled pair in spin-dependent reactions and molecular spintronics. Furthermore, quintets formed via singlet fission offer a test bed for investigating the multiexciton intermediates found in a wider range of photoinduced processes. However, many fundamental spin properties of the quintet state remain uncharacterized.

Since the quintet state has a net spin, its properties are accessible through spin-sensitive techniques: from pair-state delocalization, hyperfine or dipolar couplings, to its spin dynamics and how they vary between triplet-pair and uncoupled triplet states. Pulsed electron spin resonance is well suited to answering such questions as it provides a way of coherently separating quintet and triplet states due to their difference in multiplicity ( $S=2$ versus $S=1$ ). Because of the higher spin of the quintet state it can couple more strongly than the triplet state to an external microwave drive, and this provides a means to coherently separate and selectively address these distinct states. This is particularly powerful for nonoriented samples, such as an organic semiconductor film, where coexisting quintet and triplet spectral features will overlap due to the simultaneous presence of both these states [Fig. 1(a)] [23]. Furthermore, electron spin resonance (ESR) offers angstrom-scale sensitivity through measurement of spin-spin couplings, providing a sensitive probe of tripletpair wave functions, and enables investigation of optically 


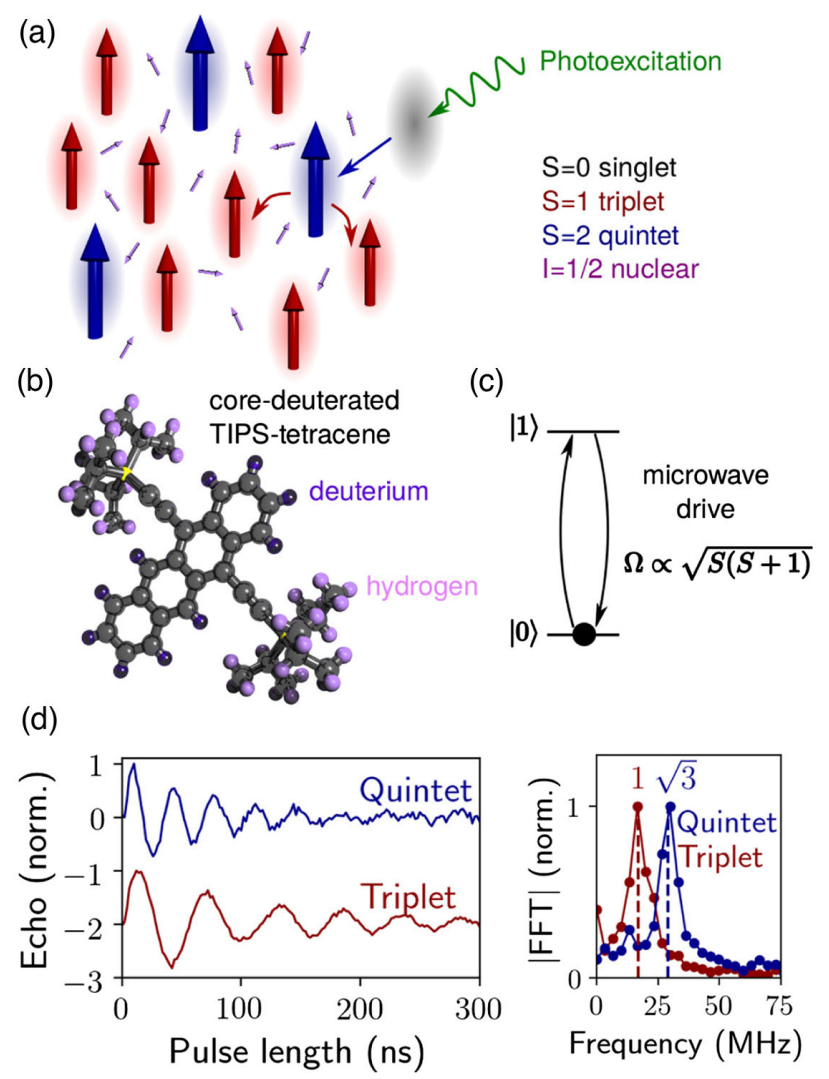

FIG. 1. Coherent spin selection of quintet and triplet states formed by singlet fission. (a) Schematic of coexisting quintet, triplet, singlet, and nuclear spin states in a singlet fission material. (b) Core-deuterated TIPS-tetracene with deuterons on the tetracene core (dark purple) and protons on the side groups (light purple) indicated. (c) Basis of coherent spin selection: the microwave drive strength $\Omega$ (the Rabi frequency) between $m=0$ and $m=1$ spin states depends on the total spin $S$, enabling quintet and triplet states to be separately addressed. (d) Rabi oscillations for quintet and triplet states in a core-deuterated TIPS-tetracene film, along with the corresponding fast Fourier transform (FFT) showing the expected factor of $\sqrt{3}$ between the nutation frequencies.

dark states which can be challenging to address through purely optical means.

Here we capitalize on the prolonged (order microseconds) spin coherence displayed by fission-borne spin states to deploy coherent spin selection and separately probe the properties of triplet-pair and triplet states. We first use this approach to separate the quintet from spectrally overlapping triplet states. We then demonstrate multiplicity specific optically generated nuclear spin polarization and extract hyperfine couplings from the quintet and triplet states to specific hydrogen sites, providing a probe of the spatial distribution of triplet-pair and triplet states.

\section{EXPERIMENTAL METHODS}

We use solution-processed films of core-deuterated TIPS-tetracene [bis(triisopropylsilylethynyl)-tetracene] in which the tetracene core is deuterated, but the TIPS side groups are hydrogenated [Fig. 1(b)]. As we discuss below, this core deuteration improves our experimental sensitivity through reducing the ESR linewidths compared to nondeuterated TIPS-tetracene, and enables the side chain protons to act as a site-specific spin label. Thin-film samples were dropcast from chloroform solution onto quartz substrates and flame sealed under helium gas in a quartz ESR tube (further sample details are contained in the Supplemental Material [24]). Pulsed ESR experiments were performed at $X$-band frequencies $(\simeq 10 \mathrm{GHz})$ using a Bruker Elexsys E-580 spectrometer. A $532 \mathrm{~nm}$ pulsed laser (5 ns pulse length) was used to photoexcite the sample, generating quintet and triplet states. Microwave manipulation was then performed after a delay time of $T_{D}$. The sample was kept at $5 \mathrm{~K}$ using a helium flow cryostat. A quasi-cw spin-1/2 and resonator impurity signal were subtracted by using the ESR signal at long times after the laser excitation where quintet and triplet states were not present.

\section{RESULTS AND DISCUSSION}

\section{A. Quintet-selective spin manipulation}

Pulsed ESR allows for measurement of the spectrum, dynamics, and localization (via dipolar and hyperfine coupling parameters) of spin species. A key asset of this technique is that the transition strength between two spin sublevels will vary depending on the total spin $S$ of the species, providing a powerful way to separate different states [25-29]. Specifically, for an ac magnetic drive between two spin sublevels $m \leftrightarrow m \pm 1$, the drive strength (the Rabi frequency) is

$$
\Omega=\omega_{1} \sqrt{S(S+1)-m(m \pm 1)},
$$

where $\omega_{1}=g \mu_{B} B_{1} / \hbar$, with $\mu_{B}$ the Bohr magneton, $g$ the electronic $g$ factor, and $B_{1}$ the ac magnetic-field strength from the microwave pulse. Here we probe $m=0 \leftrightarrow m=$ \pm 1 transitions for quintet and triplet states for which Eq. (1) reduces to $\Omega=\omega_{1} \sqrt{S(S+1)}$ [Fig. 1(c)], meaning the driving strength seen by a quintet state $(S=2)$ will be $\sqrt{3}$ times higher than a triplet state $(S=1)$. This is demonstrated in Fig. 1(d), where we show Rabi oscillations of the photoexcited quintet and triplet states for the same external microwave drive, along with their fast Fourier transform. As expected from Eq. (1), the quintet oscillation frequency is $\sqrt{3}$ times higher than the triplet (further details on the pulse sequence and additional Rabi oscillation data are shown in the Supplemental Material [24]). In this article, we use this selectivity by spin multiplicity to independently probe quintet and triplet states. 


\section{B. Separating quintet and triplet states}

As indicated in Fig. 2(c), device-relevant thin-film samples contain a distribution of microcrystalline or amorphous domains each defined by an orientation relative to the external magnetic field. This noncrystalline distribution of molecular orientations results in a distribution of resonant fields due to the orientation dependence of the electron-hole dipolar interaction (the zero-field splitting), as shown schematically in Fig. 2(d) for quintet and triplet spectra. [This zero-field splitting interaction gives an energy splitting of $g \mu_{B} B \pm \frac{3}{2} h\left[D\left(\cos ^{2} \gamma-1 / 3\right)+E\left(\cos ^{2} \alpha-\cos ^{2} \beta\right)\right]$ between the $m=0$ and $m= \pm 1$ states, where $B$ is the external field, $D$ and $E$ are the zero-field splitting parameters, and $\alpha, \beta, \gamma$ the angles between the field and the molecular $X, Y, Z$ axes.] Quintet and triplet spectra will therefore overlap, and
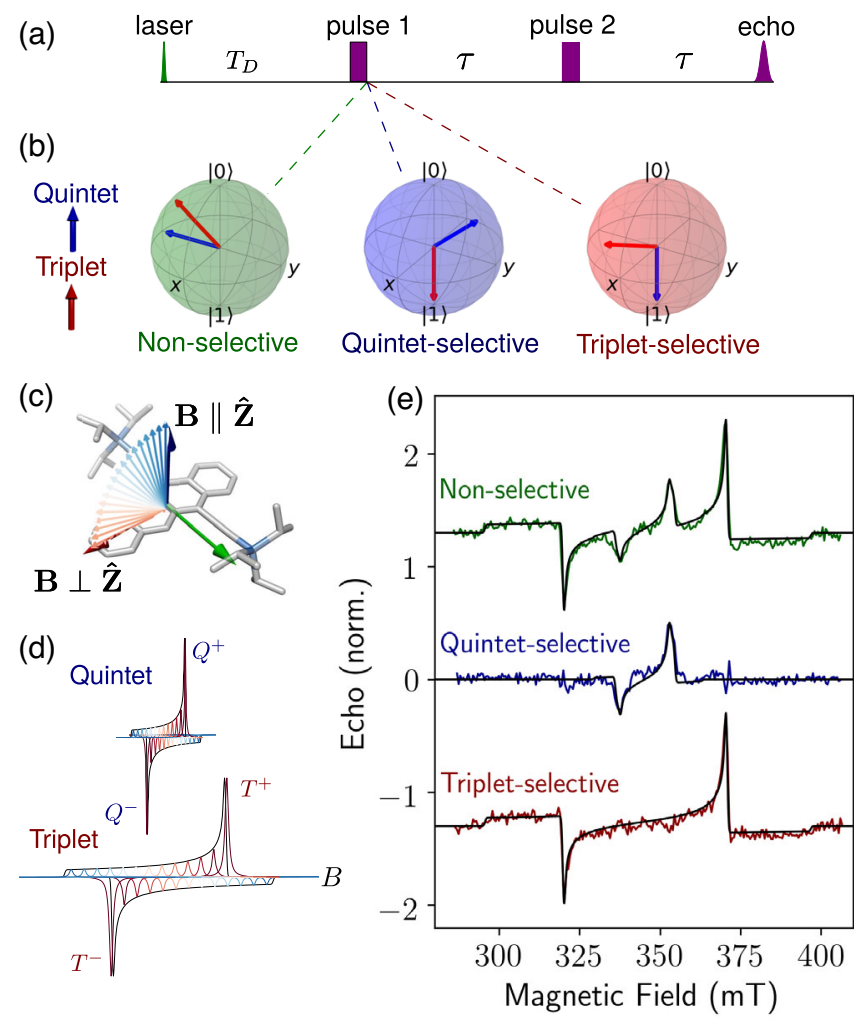

FIG. 2. Spectral separation of fission-borne spin states in a thin film. (a) Spin-echo pulse sequence. The length of pulse 1 determines which spin multiplicity is selected in the echodetected field-swept spectra by rotating triplet (red), quintet (blue), or neither state (green) onto the pole of the Bloch sphere, as depicted in (b). (c) Distribution of the magnetic field $\mathbf{B}$ relative to the molecular axes of TIPS-tetracene for a randomly oriented film with (d) corresponding ESR transitions from the $|0\rangle$ sublevel of the $S=2$ quintet states (top) and $S=1$ triplet states (bottom). (e) Spin-echo-detected spectra measured at $T_{D} \simeq 0.5 \mu \mathrm{s}$ with nonselective (green), quintet-selective (blue), and triplet-selective (red) pulse sequences, demonstrating coherent separation of quintet and triplet states. Corresponding simulations are shown in black lines. measurement of the individual properties of these states can be challenging.

However, by implementing multiplicity selective spin echoes, spectrally overlapping quintet and triplet states can be disentangled. Figure 2(e) (top) shows the spin-echo signal as a function of the external field using a standard Hahn-echo sequence, showing contributions from both quintet and triplet states. In order to separate these two components, we modify the standard Hahn-echo pulse sequence to use pulse lengths that select either the quintet or triplet state by producing an echo on the species of interest and preventing an echo from the other species [Figs. 2(a) and 2(b)]. This is readily achieved by adjusting the length of the first microwave pulse such that it inverts the spin population (a $\pi$ pulse) for the species not being measured [shown in Bloch sphere representation in Fig. 2(b)]. Because of a difference in nutation frequency, the species of interest (e.g., quintet) will develop an inplane magnetization and hence gives rise to a spin echo, while the other species (e.g., triplet) will not. This is shown experimentally in Fig. 2(e), where quintet and triplet spectra are separated via their spin multiplicity, despite the fact that the spectra from both states coexist in the same field range. [Since the quintet-selective sequence produces an echo with the opposite sign to the nonselective and triplet-selective sequences-see Fig. 2(b) -we plot the negative of the experimentally detected echo for the quintet-selective traces for clarity.] This simple approach to triplet-pair selectivity means that it can be readily applied to other spin-echo-based experiments, as we show below. We note that this coherent spectral separation would be particularly useful for systems where triplet spin polarization is much greater than that of the quintet, making triplet pairs otherwise unobservable. We now use this technique to examine the distinct properties of the quintet and triplet states.

\section{Quintet and triplet spectra}

First, we analyze the separated spectra of the quintet and triplet species [experimental spectra and simulations are shown in Fig. 2(e)]. The triplet spectrum measured following optical excitation shows a dominant $m=0$ Zeeman state population, as expected from singlet fission [24,30,31], and the spectral fit gives triplet zero-field splitting parameters $D_{T}=1.4 \mathrm{GHz}, E_{T} \leq D_{T} / 100$, consistent with previous measurements of fully hydrogenated TIPS-tetracene $[12,32,33]$. However, the line broadening is approximately 3 times narrower than in fully hydrogenated TIPS-tetracene, demonstrating a significant hyperfine line-broadening mechanism due to interaction with core protons [34] (further details on the effects of core deuteration on the ESR linewidths are contained in the Supplemental Material [24]). The corresponding quintet spectrum measured directly after optical excitation also shows an $m=0$ polarization, as described previously [12]. This quintet 
spectrum is consistent with the diabatic limit of spin polarization generation considered by Collins et al. [35] for which singlet fission predominantly populates the $m=0$ quintet state, with negligible population in the other quintet spin sublevels or the $S=1$ triplet-pair manifold (see Supplemental Material for further discussion of spin sublevel populations [24]). The isolated quintet spectrum and the lower linewidths from using core-deuterated TIPStetracene reveals that a nonzero quintet fine-structure parameter $\left(E_{Q} \neq 0\right)$ is needed to reproduce the observed line shape, in contrast to the observed axial nature of the triplet spectrum $E_{T} \simeq 0$. (In particular, this quintet nonaxiality is manifested as less sharp spectral edges at the $Q^{ \pm}$ peaks compared to the $T^{ \pm}$peaks.) We find that the combination of two noncollinear molecules from the TIPS-tetracene unit cell is not sufficient to reproduce the observed fine structure, but that the data can be explained by including an intertriplet dipolar coupling, which modifies the quintet fine structure. This is described in more detail in Ref. [36].

\section{Spin dynamics of quintet and triplet states}

We now turn to using quintet selectivity to examine the time evolution of quintet and triplet states. We capitalize on the free evolution that pulsed ESR affords to measure free spin dynamics (in contrast to previous transient ESR measurements which involve a continuous microwave field). First we use the quintet-selective sequence to measure the quintet lifetime, by varying the time between the optical excitation and the echo sequence $\left[T_{D}\right.$ in the pulse sequence shown in Fig. 2(a)]. We find a lifetime of $\tau_{Q}=17 \pm 1 \mu \mathrm{s}$ [Fig. 3(a)], demonstrating the surprisingly long-lived nature of these biexciton states. (We note that this is consistent with delayed photoluminescence and transient absorption signatures of the triplet-pair state in TIPS-tetracene which have been reported to have a $\sim 10 \mu \mathrm{s}$ lifetime in crystalline films at $10 \mathrm{~K}$ [37].)

We next examine the difference in spin coherence for the quintet and triplet states [Fig. 3(b)] by varying the free evolution time $2 \tau$ of the echo sequence. (We note that the measurements for the triplet transitions do not require a selective sequence due to the spectral separation from the quintet state.) Interestingly, we find essentially the same $T_{2}=0.75 \pm 0.02 \mu \mathrm{s}$ for quintet and triplet states. Furthermore, these values differ only slightly from nondeuterated TIPS-tetracene for which the quintet and triplet coherence times were $0.87 \pm 0.04$ and $0.69 \pm 0.04 \mu \mathrm{s}$, respectively [12]. This indicates that the core protons play only a minor role in decoherence in this system despite their significant contribution to line broadening $(\times 3$ compared to core deuterons). Such behavior is consistent with decoherence predominantly arising from fluctuating magnetic fields from the weakly coupled side-chain nuclei, with the more strongly coupled core protons serving to produce a quasistatic line broadening [38]. Modulation of the
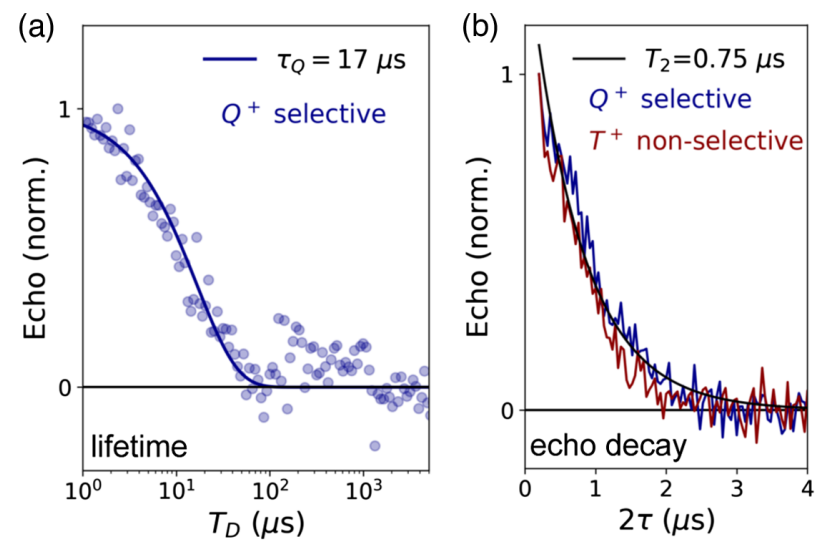

FIG. 3. Quintet spin lifetime and coherence for quintet and triplet states. (a) Selective measurement of the quintet lifetime at the $Q^{+}$transition with an exponential fit to a quintet lifetime of $\tau_{Q}=17 \pm 1 \mu \mathrm{s}$. (b) Echo decay measurement for quintet and triplet states ( $Q^{+}$and $T^{+}$transitions, respectively) along with an exponential fit with $T_{2}=0.75 \pm 0.02 \mu \mathrm{s}$.

zero-field splitting tensor by molecular vibrations or triplet exciton hopping is a further possible source of decoherence. However, we expect this mechanism to produce spectral distortions from orientation-dependent decoherence, which we do not observe $[39,40]$. A further contribution to decoherence could arise from the presence of charges, something which would be interesting to investigate in future studies through, for example, the controlled incorporation of dopants or generating quintet and triplet states in a device architecture [41,42].

While the decoherence times are indistinguishable between triplet and quintet states, the spin lifetime varies drastically between coupled and uncoupled triplet states. Unlike the quintet, the triplet polarization evolves over hundreds of microseconds reflecting the significantly longer lifetime of the triplet state (Fig. 4). As shown in Figs. 4(c) and 4(d), the initial $m=0$ fission-generated spectrum evolves to yield an $A A E E$ pattern by $T_{D}=1 \mathrm{~ms}$ (where $A$ signifies absorptive and $E$ emissive), which reflects spin population selective in the zero-field basis of the molecule $[43,44]$. This late-time spectrum is shown in Fig. 4(d) along with a simulation which demonstrates overpopulation of the zero-field $|Z\rangle$ eigenstate. The observed spectral behavior is consistent with spin-selective decay via spin-orbit mediated intersystem crossing (ISC) of the triplet excited state to the singlet ground state, which more rapidly depletes the zero-field eigenstates $|X\rangle$ and $|Y\rangle$ (further details are contained in the Supplemental Material [24]). Here we parametrize this spin-selective population decay by the ISC decay rates $k_{X Y}$ for the $|X\rangle,|Y\rangle$ states, and $k_{Z}$ for the $|Z\rangle$ state, and the spin-lattice relaxation time $T_{1}$.

As shown in Fig. 4 and described in the Supplemental Material [24], at the $T_{\|}^{+}$transition [Fig. 2(c), B $\| \hat{\mathbf{Z}}$ ] the $|0\rangle$ and $|1\rangle$ levels have decay rates $k_{Z}$ and $k_{X Y}$, respectively, and so the initial overpopulation of $|0\rangle$ is enhanced by the 

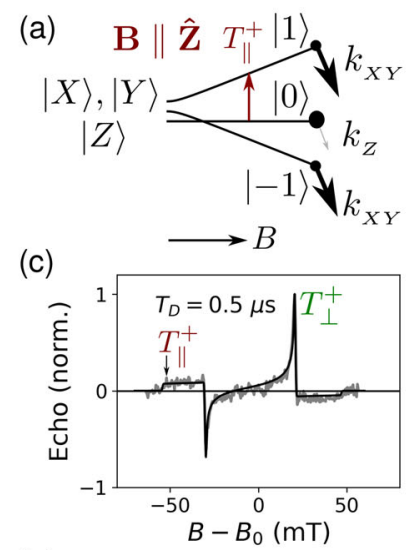

(b) $\mathbf{B} \perp \hat{\mathbf{Z}}_{T^{+}}|1\rangle$.

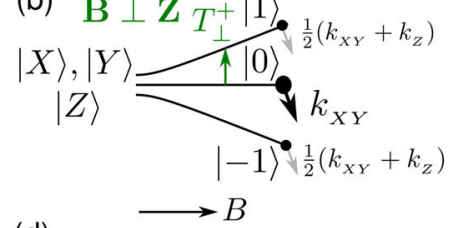

(d)

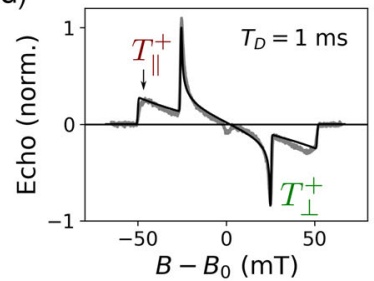

(6)

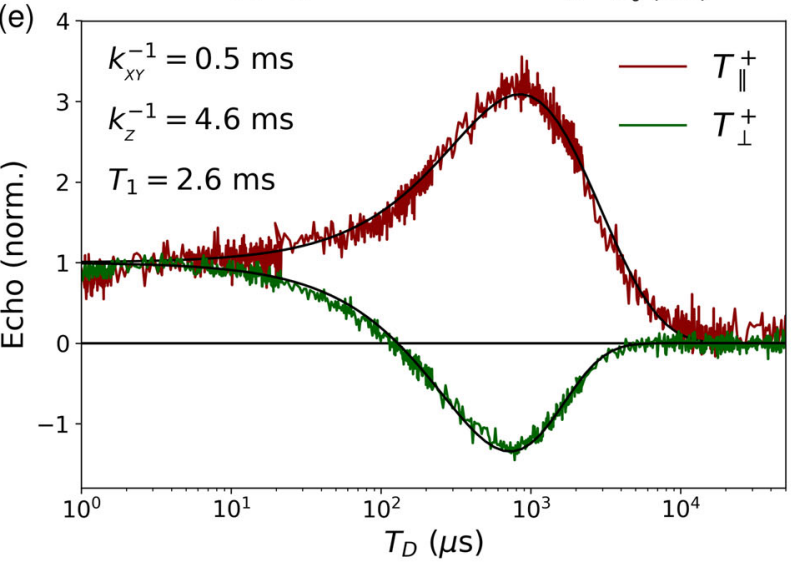

FIG. 4. Spin-orbit mediated decay of uncoupled triplet excitons formed by singlet fission. (a) Spin sublevel decay schematic for $\mathbf{B} \| \hat{\mathbf{Z}}$ with a decay rate of $k_{Z}$ for the $|0\rangle$ state and $k_{X Y}$ for the $| \pm 1\rangle$ states. (b) Decay schematic for $\mathbf{B} \perp \hat{\mathbf{Z}}$ with a decay rate of $k_{X Y}$ for the $|0\rangle$ state and $\frac{1}{2}\left(k_{X Y}+k_{Z}\right)$ for the $| \pm 1\rangle$ states. (c),(d) Echodetected spectra (gray) with $T_{D}=1 \mu \mathrm{s}$ and $T_{D}=1 \mathrm{~ms}$ along with simulations (black) for overpopulation of the Zeeman $|0\rangle$ state, and the zero-field $|Z\rangle$ state, respectively. (e) Experimental lifetime measurements for the $T_{\|}^{+}$and $T_{\perp}^{+}$field positions shown in (c) and (d), along with a fit giving $k_{X Y}^{-1}=0.51 \pm 0.03 \mathrm{~ms}, k_{Z}^{-1}=$ $4.6 \pm 0.2 \mathrm{~ms}$, and spin-lattice relaxation time $T_{1}=2.6 \pm 0.1 \mathrm{~ms}$.

longer lifetime of this state relative to the $|1\rangle$ state. By contrast, at the $T_{\perp}^{+}$[Fig. 2(c), $\mathbf{B} \perp \hat{\mathbf{Z}}$ ] transition the $|0\rangle$ and $|1\rangle$ levels have decay rates $k_{X Y}$ and $\frac{1}{2}\left(k_{X Y}+k_{Z}\right)$, respectively, and so the initial polarization becomes inverted due to the longer lifetime of $|1\rangle$ relative to $|0\rangle$. Using a global fit for the two time traces we extract the spin-orbit mediated decay rates $k_{Z}^{-1}=4.6 \pm 0.2 \mathrm{~ms}$ and $k_{X Y}^{-1}=0.51 \pm 0.03 \mathrm{~ms}$ and the spin-lattice relaxation time of $T_{1}=2.6 \pm 0.1 \mathrm{~ms}$. (We note that the fit is constrained to start after the rise and equilibration of the initial spin population by singlet fission and that these measurements do not require multiplicity selective pulses due to spectral separation from the quintet state.)

These measurements point to the key role of triplettriplet coupling in pathways for spin-state decay of the quintet state: the quintet lifetime is significantly shorter than that of the triplet state. This suggests that the dominant loss pathway for pair states is not intersystem crossing of the underlying triplet excitons to the ground state but rather pair dissociation or recombination. We note that recombination of the quintet state would require spin mixing with the triplet and singlet pair states, for which triplet-triplet annihilation is spin allowed, and points to a key question for future investigation into how such mixing occurs. The enhanced relaxation and decay of the quintet relative to the triplet suggests that exciton recombination, dissociation, and spin-spin coupling are key parameters for future optimization of the spin properties of the multiexciton state. Having discussed the properties of the electronic spin states involved in singlet fission, we now turn to their interaction with nuclear spins in their surrounding environment.

\section{E. Multiplicity selective coherent nuclear coupling via spin locking}

Measurement of the hyperfine couplings of a state provides a highly sensitive probe of its wave function $[45,46]$, and are thus important to access for triplet-pair states. In the presence of coexisting triplet states, it is important to be able to selectively address the quintet state's hyperfine interactions as well as use a technique which is compatible with the relatively short-lived nature of the biexciton (compared to typical nuclear spin manipulation times). An attractive approach to meet both of these requirements is to use purely electron spin manipulation and interrogate hyperfine coupling through its effect on the electronic spin state. Importantly, this can readily be applied in a multiplicity selective way through a spinlocking sequence, also referred to as nuclear orientation via electron spin locking $[47,48]$, allowing separation of the nuclear coupling of quintet and triplet states.

The principle of this approach and its corresponding pulse sequence is shown in Figs. 5 and 6(a) $[47,48]$. The basic idea is to overcome the difference in energy splittings between electronic and nuclear spin states by generating a superposition state of the electronic spin (a dressed state) whose energy is determined by the microwave Rabi frequency $\Omega$, rather than the static Zeeman energy and zero-field splitting. This dressed state can then be brought into resonance with the nuclear spin splitting, enabling electronic spin polarization to be transferred to nuclear spin polarization. Briefly, a quintet (or triplet) spin initially polarized along the $z$ direction is rotated onto the $-y$ axis in the rotating frame by a microwave $\pi / 2$ pulse oriented along the $x$ axis. (Here the $z$ axis is defined by the static magnetic field, the $x$ axis by the microwave drive, and the $y$ axis by the mutually perpendicular direction.) This is immediately followed by a $90^{\circ}$ phase-shifted pulse (which corresponds to a pulse polarized along the $y$ axis), which locks the spin along the $-y$ axis. Under this spin-locking condition the spin sees a static magnetic field $B_{1}$ along this axis. The eigenstates of this system are then the dressed states $|+\rangle$, 
(a)

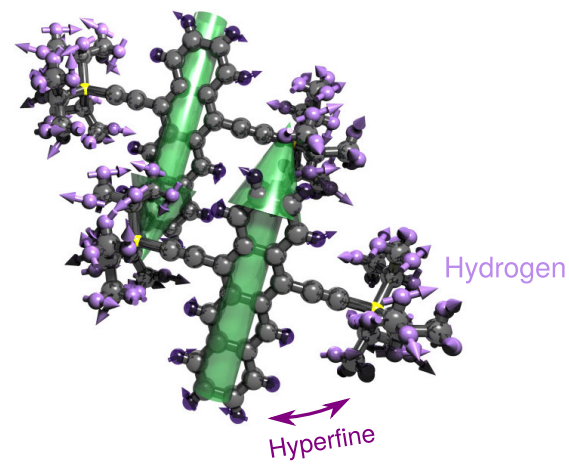

(b) Electron-nuclear coupling



FIG. 5. State- and site-selective electron-nuclear coupling in a singlet fission material. (a) Selectively deuterated TIPS-tetracene with photoexcited triplet pair (green) showing coupling to hydrogen nuclear spins on the side groups. (b) Schematic of electron-nuclear coupling under spin-locking conditions showing microwave driving to form dressed states with a Rabi splitting matching the $\sim 10 \mathrm{MHz}$ nuclear spin splitting, allowing coherent electron-nuclear evolution and polarization transfer. $|-\rangle$, which are now quantized along the microwave field with energies $\pm \frac{1}{2} \hbar \omega_{1} \sqrt{S(S+1)}$.

When this energy splitting matches the splitting between nuclear states (the $m= \pm \frac{1}{2}$ nuclear states $|\uparrow\rangle,|\downarrow\rangle$ ), the $|+\downarrow\rangle$ and $|-\uparrow\rangle$ states can coherently mix in the presence of hyperfine interactions. Under this matching condition, termed Hartmann-Hahn resonance [49], the polarization evolves coherently, oscillating between the electrons and nuclei with a frequency that depends on the hyperfine coupling. This hyperfine-induced coherent evolution thereby provides a way to measure the hyperfine coupling in these states. Since the dressed-state energy splitting is dependent on the spin multiplicity $[\Omega \propto \sqrt{S(S+1)}]$, quintet and triplet states can be separately addressed. Furthermore, since the electron spin is initially polarized due to fission, the $|-\rangle$ dressed state will predominantly be populated [Fig. 5(b)] and transfer from $|-\uparrow\rangle \rightarrow|+\downarrow\rangle$ therefore transfers the electron spin polarization to initially unpolarized nuclei, optically generating a net nuclear spin polarization [48,50,51].

Figures 6(b) and 6(c) show the echo intensity as a function of the locking pulse length at the quintet $\left(Q^{+}\right)$and triplet $\left(T^{+}\right)$transitions under resonant matching conditions $\sqrt{S(S+1)} \omega_{1}=\omega_{H}$ as well as detuned from this condition at $\sqrt{S(S+1)} \omega_{1}=\omega_{H} / 2$, where $\omega_{H}$ is the Larmor frequency of the hydrogen nuclear spins on the side chains. We note that since the deuteron Larmor frequency is 6.5 times smaller than for the proton [52], the core deuterons

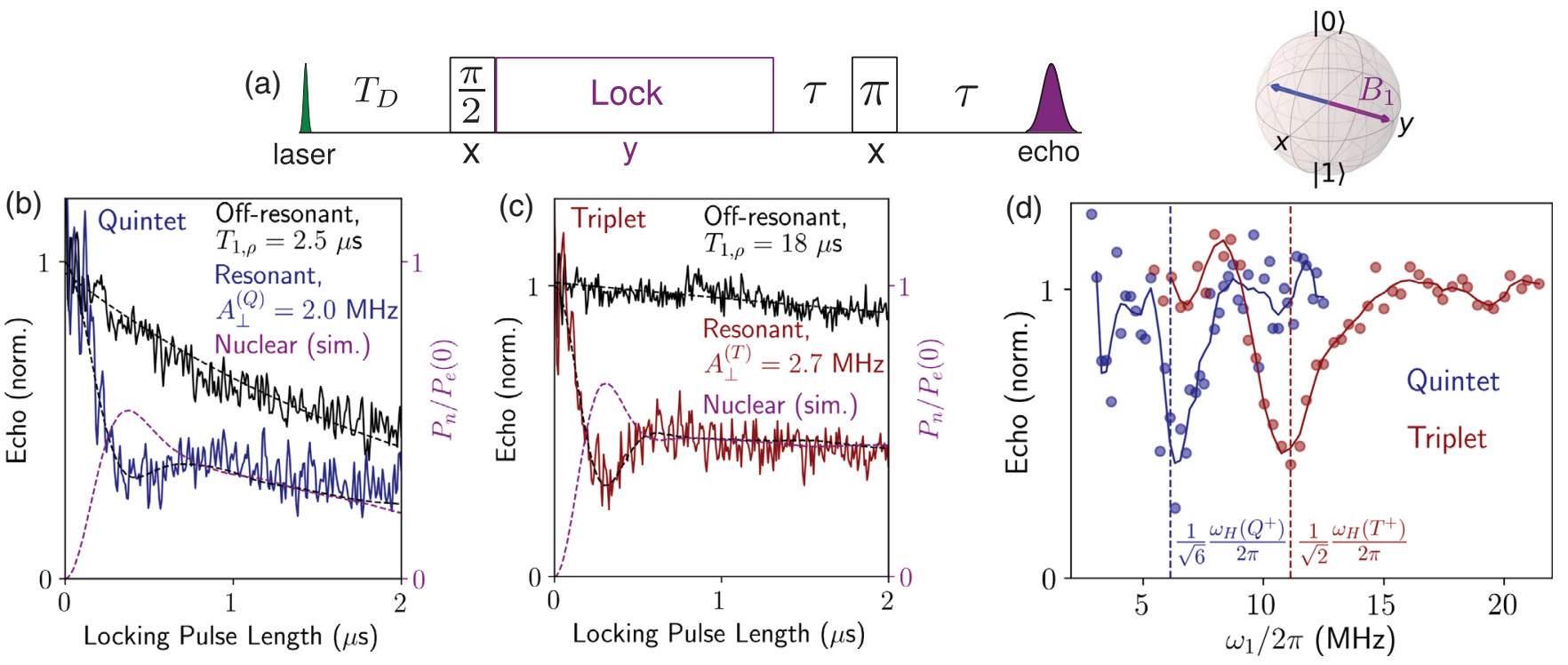

FIG. 6. Selectively coupling quintet and triplet states to site-specific nuclei. (a) Pulse sequence for spin polarization transfer from electronic to nuclear degrees of freedom along with Bloch sphere depiction of spin locking. (b),(c) Pulse-length-dependent polarization transfer from quintet and triplet states to side-chain nuclear spins with simulations for electronic $\left(P_{e}\right)$ and nuclear $\left(P_{n}\right)$ spin polarization shown in dashed lines, with $P_{e}(0)$ the initial electronic spin polarization. (d) Multiplicity selective polarization transfer shown as a function of the microwave driving field $\left(\omega_{1}\right)$ for quintet (400 ns locking time) and triplet states (300 ns locking time) measured at the $Q^{+}$ and $T^{+}$transitions, respectively. 
would be brought into resonance at much lower frequencies $(\simeq 2 \mathrm{MHz})$ and are therefore decoupled from the response of the side-group proton spins, which provide a label to selectively address the hyperfine coupling to the side chains.

Detuned from resonance, the spins are locked along the $-y$ axis by the microwave field and decay with their respective rotating frame relaxation times $T_{1, \rho}$, which we find to be $18 \mu \mathrm{s}\left(T^{+}\right)$and $2.5 \mu \mathrm{s}\left(Q^{+}\right)$. At Hartmann-Hahn resonance, however, a very different behavior arises. Now the dressed spins are resonantly coupled with surrounding hydrogen nuclei and can coherently mix at a rate determined by their hyperfine coupling. The echo intensity will then undergo oscillations at the coupling frequency as polarization is exchanged between electrons and nuclei. Damped oscillations, as we observe here, arise when there is a distribution of coupling strengths, which we expect due to the presence of multiple inequivalent hydrogen nuclei [Fig. 5(a)] and from the ensemble averaging of molecules within the film.

The hyperfine coupling is read out in the oscillatory driven electron spin, which acts as a sensor of the local nuclear environment, a scheme for quantum metrology demonstrated with external quantum sensors [51] and here with the intrinsic spin states generated by singlet fission. We simulate the polarization transfer dynamics in Figs. 6(b) and 6(c) by finding the density-matrix evolution of a quintet or triplet coupled to a hydrogen nuclear spin, averaging over a Gaussian distribution of hyperfine couplings with center $A_{\perp}$ and standard deviation $\sigma_{A_{\perp}}$ (simulation details are contained in the Supplemental Material [24]). The resulting traces show good agreement with the experimental data with $A_{\perp}^{(Q)}=2.0 \mathrm{MHz}, \sigma_{A_{\perp}}^{(Q)}=1.2 \mathrm{MHz}$ (quintet) and $A_{\perp}^{(T)}=2.7 \mathrm{MHz}, \sigma_{A_{\perp}}^{(T)}=1.3 \mathrm{MHz}$ (triplet). Figures 6(b) and 6(c) also show the corresponding simulated nuclear polarization $P_{n}$ normalized to the initial quintet or triplet polarization $P_{e}(0)$. These traces show a maximum polarization transfer of $\simeq 70 \%$ (triplet, 400 ns locking pulse) and $\simeq 50 \%$ (quintet, $300 \mathrm{~ns}$ locking pulse). By further reducing the distribution of hyperfine couplings, this polarization transfer could be increased further. Future work incorporating simultaneous ESR and nuclear magnetic resonance detection would further provide an exciting opportunity to measure and optimize the bulk nuclear spin polarization generated by singlet fission. While optically generated polarization from intersystem crossing has been established as a promising platform for dynamic nuclear polarization in isolated molecules [53-55] as well as spin defects in solids [50,56-58], to our knowledge, this is the first demonstration of dynamic nuclear spin polarization from singlet fission.

Because of the higher total spin, the quintet $|0\rangle \leftrightarrow|1\rangle$ transition $\left(Q^{+}\right)$has a $\sqrt{3}$ higher Rabi frequency compared to the triplet $|0\rangle \leftrightarrow|1\rangle$ transition $\left(T^{+}\right)$, meaning that quintet states are resonantly coupled to nuclei at physically separate $\omega_{1}$ driving fields. This is more clearly demonstrated in Fig. 6(d), where we fix the locking pulse length and sweep its power, i.e., $\omega_{1}$. $(\pi / 2$ and $\pi$ pulses are adjusted according to the Rabi frequency, and since the echo intensity also increases with $\omega_{1}$, we normalize by a linear baseline for clarity.) Because of their difference in total spin, the $Q^{+}$and $T^{+}$transitions display multiplicity selective resonances, with the expected factor of $\sqrt{3}$ between their positions: the higher spin of the quintet state means Hartmann-Hahn matching is achieved for lower driving powers than for the triplet. Similarly, the higher spin of the quintet means it could be more readily matched to the nuclear Zeeman energy at higher magnetic fields than lower spin states [59].

The hydrogen spins present only on the TIPS side groups act as a spin label which provide information on the degree of delocalization of the quintet or triplet wave functions onto these side chains. The extracted hyperfine coupling strengths correspond to electron-nuclear dipolar coupling distances of $\sim 3 \AA$, which is approximately the distance between the tetracene core and the TIPS protons, suggesting that spin density is mainly localized on the tetracene core. For comparison, we measured hyperfine couplings at the $T^{+}$transition in fully protonated TIPS-tetracene through electron-nuclear double resonance, which is feasible for the much longer-lived triplet states, where we found couplings up to $6 \mathrm{MHz}$ (see Supplemental Material [24]), much larger than those measured in Fig. 6(c), which we assign to the stronger interactions with protons on the tetracene core. Interestingly, we find a weaker hyperfine coupling for the quintet state than the triplet, which could be explained by averaging over a greater number of nuclear spins in a tripletpair state compared to a single triplet state due to increased delocalization.

\section{CONCLUSION}

From measurement of relaxation and spin-selective decay to hyperfine coupling and nuclear polarization, coherent control of singlet fission generated spin states opens up a wealth of methods to interrogate their key properties. Our measurements reveal that while spin coherence times $\left(T_{2}\right)$ are similar in both quintet and triplet states, the spin lifetimes are reduced by over 2 orders of magnitude in the quintet state compared to the triplet state. This enhanced quintet relaxation indicates that intertriplet coupling determines the fate of the biexciton, and points to the need for synthetic control of this property for prolonged biexciton spin lifetimes. The pure triplet state, on the other hand, with its millisecond relaxation times, may find a niche in spin-based sensors with facile fabrication, low cost, and material flexibility [60].

Beyond probing dynamic properties, coherent control enables investigation of the hyperfine coupling associated with triplet-exciton pairs and allows independent coherent 
coupling of photoexcited quintet and triplet states to nuclei within the same material. In doing so we have demonstrated optical polarization of nuclear spins with singlet exciton fission for the first time and present a pathway for mapping the quintet state via hyperfine coupling. Future experiments selectively deuterating different points around the molecule would further allow site-specific hyperfine interactions to be uncovered. In contrast to intersystem crossing, which relies on spin-orbit coupling and is hence relatively slow to induce electron spin polarization (of order milliseconds; see Fig. 4), singlet fission is intriguing in offering polarization through spin-dependent reactions, and hence can be much faster in generating initial electron spin polarization than intersystem crossing. Furthermore, since spin polarization by singlet fission does not rely on thermal statistics, fissiongenerated dynamic nuclear polarization should be possible at room temperature and a simple kinetic model indicates that weakly coupled triplets can be generated with up to 33\% spin polarization (see Supplemental Material [24]). More broadly, dynamically polarizing nuclei in organic semiconductors should also be possible with the spinpolarized electron-hole pairs which form in organic photovoltaic or light-emitting materials [61], providing a pathway to map the solid-state environment of excited states in optoelectronic materials through targeted polarization transfer. Along with optical or electrical readout schemes [62-64], coherent manipulation of fissiongenerated spins provides a promising way to uncover the influence of spin-spin coupling and nuclear spins in organic semiconductor materials, and opens up new possibilities for organic spintronics $[65,66]$.

\section{ACKNOWLEDGMENTS}

We thank C. Teutloff and R. Steyrleuthner for experimental assistance, and A. D. Chepelianskii, R. H. Friend, and N. Panjwani for helpful comments. We acknowledge support from DFG SPP-1601 (Bi-464/10-2 and BE-5126/ 1-2). L. R. W. acknowledges support from the Clare College Junior Research Fellowship.

[1] B. D. Ravetz, A. B. Pun, E. M. Churchill, D. N. Congreve, T. Rovis, and L. M. Campos, Photoredox Catalysis Using Infrared Light via Triplet Fusion Upconversion, Nature (London) 565, 343 (2019).

[2] T. N. Singh-Rachford and F. N. Castellano, Photon Upconversion Based on Sensitized Triplet-Triplet Annihilation, Coord. Chem. Rev. 254, 2560 (2010).

[3] J. Mezyk, R. Tubino, A. Monguzzi, A. Mech, and F. Meinardi, Effect of an External Magnetic Field on the Up-Conversion Photoluminescence of Organic Films: The Role of Disorder in Triplet-Triplet Annihilation, Phys. Rev. Lett. 102, 087404 (2009).

[4] R. Liu, Y. Zhang, Y. L. Lei, P. Chen, and Z. H. Xiong, Magnetic Field Dependent Triplet-Triplet Annihilation in
$\mathrm{Alq}_{3}$-Based Organic Light Emitting Diodes at Different Temperatures, J. Appl. Phys. 105, 093719 (2009).

[5] H. van Eersel, P. A. Bobbert, and R. Coehoorn, Kinetic Monte Carlo Study of Triplet-Triplet Annihilation in Organic Phosphorescent Emitters, J. Appl. Phys. 117, 115502 (2015).

[6] M. B. Smith and J. Michl, Singlet Fission, Chem. Rev. 110, 6891 (2010).

[7] M. B. Smith and J. Michl, Recent Advances in Singlet Fission, Annu. Rev. Phys. Chem. 64, 361 (2013).

[8] D. N. Congreve, J. Lee, N. J. Thompson, E. Hontz, S. R. Yost, P. D. Reusswig, M. E. Bahlke, S. Reineke, T. Van Voorhis, and M. A. Baldo, External Quantum Efficiency above $100 \%$ in a Singlet-Exciton-Fission-Based Organic Photovoltaic Cell, Science 340, 334 (2013).

[9] N. J. Thompson, M. W. B. Wilson, D. N. Congreve, P. R. Brown, J. M. Scherer, T. S. Bischof, M. Wu, N. Geva, M. Welborn, T. Van Voorhis, V. Bulovic, M. G. Bawendi, and M. A. Baldo, Energy Harvesting of Non-Emissive Triplet Excitons in Tetracene by Emissive PbS Nanocrystals, Nat. Mater. 13, 1039 (2014).

[10] M. Tabachnyk, B. Ehrler, S. Gélinas, M. L. Böhm, B. J. Walker, K. P. Musselman, N. C. Greenham, R. H. Friend, and A. Rao, Resonant Energy Transfer of Triplet Excitons from Pentacene to PbSe Nanocrystals, Nat. Mater. 13, 1033 (2014).

[11] M. Einzinger, T. Wu, J. F. Kompalla, H. L. Smith, C. F. Perkinson, L. Nienhaus, S. Wieghold, D. N. Congreve, A. Kahn, M. G. Bawendi, and M. A. Baldo, Sensitization of Silicon by Singlet Exciton Fission in Tetracene, Nature (London) 571, 90 (2019).

[12] L. R. Weiss, S. L. Bayliss, F. Kraffert, K. J. Thorley, J. E. Anthony, R. Bittl, R. H. Friend, A. Rao, N. C. Greenham, and J. Behrends, Strongly Exchange-Coupled Triplet Pairs in an Organic Semiconductor, Nat. Phys. 13, 176 (2017).

[13] M. J. Y. Tayebjee, S. N. Sanders, E. Kumarasamy, L. M. Campos, M. Y. Sfeir, and D. R. McCamey, Quintet Multiexciton Dynamics in Singlet Fission, Nat. Phys. 13, 182 (2017).

[14] B. S. Basel, J. Zirzlmeier, C. Hetzer, B. T. Phelan, M. D. Krzyaniak, S. R. Reddy, P. B. Coto, N. E. Horwitz, R. M. Young, F. J. White, F, Hampel, T. Clark, M. Thoss, R. R. Tykwinski, M. R. Wasielewski, and D. M. Guldi, Unified Model for Singlet Fission within a Non-Conjugated Covalent Pentacene Dimer, Nat. Commun. 8, 15171 (2017).

[15] D. Lubert-Perquel, E. Salvadori, M. Dyson, P. N. Stavrinou, R. Montis, H. Nagashima, Y. Kobori, S. Heutz, and C. W. M. Kay, Identifying Triplet Pathways in Dilute Pentacene Films, Nat. Commun. 9, 4222 (2018).

[16] H. Sakai, R. Inaya, H. Nagashima, S. Nakamura, Y. Kobori, N. V. Tkachenko, and T. Hasobe, Multiexciton Dynamics Depending on Intramolecular Orientations in Pentacene Dimers: Recombination and Dissociation of Correlated Triplet Pairs, J. Phys. Chem. Lett. 9, 3354 (2018).

[17] H. Nagashima, S. Kawaoka, S. Akimoto, T. Tachikawa, Y. Matsui, H. Ikeda, and Y. Kobori, Singlet-Fission-Born Quintet State: Sublevel Selections and Trapping by Multiexciton Thermodynamics, J. Phys. Chem. Lett. 9, 5855 (2018). 
[18] Y. Matsui, S. Kawaoka, H. Nagashima, T. Nakagawa, N. Okamura, T. Ogaki, E. Ohta, S. Akimoto, A. Sato-Tomita, S. Yagi, Y. Kobori, and H. Ikeda, Exergonic Intramolecular Singlet Fission of an Adamantane-Linked Tetracene Dyad via Twin Quintet Multiexcitons, J. Phys. Chem. C 123, 18813 (2019).

[19] A.B. Pun, A. Asadpoordarvish, E. Kumarasamy, M. J. Y. Tayebjee, D. Niesner, D. R. McCamey, S. N. Sanders, L. M. Campos, and M. Y. Sfeir, Ultra-Fast Intramolecular Singlet Fission to Persistent Multiexcitons by Molecular Design, Nat. Chem. 11, 821 (2019).

[20] V. I. Klimov, J. A. McGuire, R. D. Schaller, and V. I. Rupasov, Scaling of Multiexciton Lifetimes in Semiconductor Nanocrystals, Phys. Rev. B 77, 195324 (2008).

[21] M. Chen, M. D. Krzyaniak, J. N. Nelson, Y. J. Bae, S. M. Harvey, R. D. Schaller, R. M. Young, and M. R. Wasielewski, Quintet-Triplet Mixing Determines the Fate of the Multiexciton State Produced by Singlet Fission in a Terrylenediimide Dimer at Room Temperature, Proc. Natl. Acad. Sci. U.S.A. 116, 8178 (2019).

[22] B. Dick and B. Nickel, Accessibility of the Lowest Quintet State of Organic Molecules through Triplet-Triplet Annihilation; An Indo Ci Study, Chem. Phys. 78, 1 (1983).

[23] J. A. Weil and J. R. Bolton, Electron Paramagnetic Resonance (Wiley, Hoboken, NJ, 2007), Chap. 6, pp. 162-187.

[24] See Supplemental Material at http://link.aps.org/ supplemental/10.1103/PhysRevX.10.021070 for further details of samples, spin sublevel populations, role of deuteration, quintet and triplet Rabi oscillations, spin-orbit decay kinetics, nuclear polarization transfer, simulations, electron-nuclear double resonance, and spin-polarization of weakly coupled triplets.

[25] A. V. Astashkin and A. Schweiger, Electron-Spin Transient Nutation: A New Approach to Simplify the Interpretation of ESR Spectra, Chem. Phys. Lett. 174, 595 (1990).

[26] L. Kevan and M. K. Bowman, Modern Pulsed and Continuous-Wave Electron Spin Resonance (WileyInterscience, New York, 1990).

[27] R. Hanaishi, Y. Ohba, K. Akiyama, S. Yamauchi, and M. Iwaizumi, Observation of Spin-Correlated Radical Pairs via Two-Dimensional Electron Paramagnetic Resonance Nutation Spectroscopy, J. Chem. Phys. 103, 4819 (1995).

[28] T. Takui, K. Sato, D. Shiomi, K. Itoh, T. Kaneko, E. Tsuchida, and H. Nishide, Ft Pulsed ESR/Electron Spin Transient Nutation (ESTN) Spectroscopy Applied to HighSpin Systems in Solids; Direct Evidence of a Topologically Controlled High-Spin Polymer as Models for Quasi ID Organic Ferro- and Superpara-Magnets, Mol. Cryst. Liq. Cryst. 279, 155 (1996).

[29] S. Stoll, G. Jeschke, M. Willer, and A. Schweiger, NutationFrequency Correlated EPR Spectroscopy: The PEANUT Experiment, J. Magn. Reson. 130, 86 (1998).

[30] L. Yarmus, J. Rosenthal, and M. Chopp, EPR of Triplet Excitions in Tetracene Crystals: Spin Polarization and the Role of Singlet Exciton Fission, Chem. Phys. Lett. 16, 477 (1972).

[31] C. E. Swenberg, R. Van Metter, and M. Ratner, Comments on Exciton Fission and Electron Spin Resonance in Tetracene Single Crystals, Chem. Phys. Lett. 16, 482 (1972).
[32] S. L. Bayliss, A. D. Chepelianskii, A. Sepe, B. J. Walker, B. Ehrler, M. J. Bruzek, J. E. Anthony, and N. C. Greenham, Geminate and Nongeminate Recombination of Triplet Excitons Formed by Singlet Fission, Phys. Rev. Lett. 112, 238701 (2014).

[33] S. L. Bayliss, K. J. Thorley, J. E. Anthony, H. Bouchiat, N. C. Greenham, and A. D. Chepelianskii, Localization Length Scales of Triplet Excitons in Singlet Fission Materials, Phys. Rev. B 92, 115432 (2015).

[34] T. D. Nguyen, G. Hukic-Markosian, F. Wang, L. Wojcik, X.-G. Li, E. Ehrenfreund, and Z. V. Vardeny, Isotope Effect in Spin Response of $\pi$-Conjugated Polymer Films and Devices, Nat. Mat. 9, 345-352 (2010).

[35] M. I. Collins, D. R. McCamey, and M. J. Y. Tayebjee, Fluctuating Exchange Interactions Enable Quintet Multiexciton Formation in Singlet Fission, J. Chem. Phys. 151, 164104 (2019).

[36] K. M. Yunusova, S. L. Bayliss, T. Cheneliere, V. Derkach, J. E. Anthony, A. D. Chepelianskii, and L. R. Weiss, Spin Fine-Structure Reveals Bi-Exciton Geometry in an Organic Semiconductor, arXiv:1912.04191.

[37] H. L. Stern, A. Cheminal, S. R. Yost, K. Broch, S. L. Bayliss, K. Chen, M. Tabachnyk, K. Thorley, N. Greenham, J. M. Hodgkiss, J. Anthony, M. Head-Gordon, A. J. Musser, A. Rao, and R. H. Friend, Vibronically Coherent Ultrafast Triplet-Pair Formation and Subsequent Thermally Activated Dissociation Control Efficient Endothermic Singlet Fission, Nat. Chem. 9, 1205 (2017).

[38] M. J. Graham, C.-J. Yu, M. D. Krzyaniak, M. R. Wasielewski, and D.E. Freedman, Synthetic Approach to Determine the Effect of Nuclear Spin Distance on Electronic Spin Decoherence, J. Am. Chem. Soc. 139, 3196 (2017).

[39] C. W. M. Kay, G. Elger, and K. Möbius, The Photoexcited Triplet State of Free-Base Porphycene: A Time-Resolved EPR and Electron Spin Echo Investigation, Phys. Chem. Chem. Phys. 1, 3999 (1999).

[40] A. Barbon, M. Bortolus, M. Brustolon, A. Comotti, A. L. Maniero, U. Segre, and P. Sozzani, Dynamics of the Triplet State of a Dithiophene in Different Solid Matrixes Studied by Transient and Pulse EPR Techniques, J. Phys. Chem. B 107, 3325 (2003).

[41] N. J. Thompson, E. Hontz, D. N. Congreve, M. E. Bahlke, S. Reineke, T. Van Voorhis, and M. A. Baldo, Nanostructured Singlet Fission Photovoltaics Subject to TripletCharge Annihilation, Adv. Mater. 26, 1366 (2014).

[42] W. J. Baker, D. R. McCamey, K. J. Van Schooten, J. M. Lupton, and C. Boehme, Differentiation between PolaronPair and Triplet-Exciton Polaron Spin-Dependent Mechanisms in Organic Light-Emitting Diodes by Coherent Spin Beating, Phys. Rev. B 84, 165205 (2011).

[43] D. E. Budil and M. C. Thurnauer, The Chlorophyll Triplet State as a Probe of Structure and Function in Photosynthesis, Biochim. Biophys. Acta 1057, 1 (1991).

[44] F. Kraffert, R. Steyrleuthner, S. Albrecht, D. Neher, M. C. Scharber, R. Bittl, and J. Behrends, Charge Separation in PCPDTBT:PCBM Blends from an EPR Perspective, J. Phys. Chem. C 118, 28482 (2014).

[45] F. Lendzian, R. Bittl, A. Telfer, and W. Lubitz, Hyperfine Structure of the Photoexcited Triplet State ${ }^{3} \mathrm{P} 680$ in Plant 
PS II Reaction Centres as Determined by Pulse ENDOR Spectroscopy, Biochim. Biophys. Acta 1605, 35 (2003).

[46] C. E. Tait, P. Neuhaus, H. L. Anderson, and C. R. Timmel, Triplet State Delocalization in a Conjugated Porphyrin Dimer Probed by Transient Electron Paramagnetic Resonance Techniques, J. Am. Chem. Soc. 137, 6670 (2015).

[47] H. Brunner, R. H. Fritsch, and K. H. Hausser, Cross Polarization in Electron Nuclear Double Resonance by Satisfying the Hartmann-Hahn Condition, Z. Naturforsch. A 42, 1456 (1987).

[48] A. Henstra, P. Dirksen, J. Schmidt, and W. T. Wenckebach, Nuclear Spin Orientation via Electron Spin Locking (Novel), J. Magn. Reson. 77, 389 (1988).

[49] S. R. Hartmann and E. L. Hahn, Nuclear Double Resonance in the Rotating Frame, Phys. Rev. 128, 2042 (1962).

[50] P. London, J. Scheuer, J.-M. Cai, I. Schwarz, A. Retzker, M. B. Plenio, M. Katagiri, T. Teraji, S. Koizumi, J. Isoya, R. Fischer, L. P. McGuinness, B. Naydenov, and F. Jelezko, Detecting and Polarizing Nuclear Spins with Double Resonance on a Single Electron Spin, Phys. Rev. Lett. 111, 067601 (2013).

[51] M. Loretz, T. Rosskopf, and C. L. Degen, Radio-Frequency Magnetometry Using a Single Electron Spin, Phys. Rev. Lett. 110, 017602 (2013).

[52] J. M. B. Kellogg, I. I. Rabi, N. F. Ramsey, Jr., and J. R. Zacharias, The Magnetic Moments of the Proton and the Deuteron. the Radiofrequency Spectrum of $\mathrm{H}_{2}$ in Various Magnetic Fields, Phys. Rev. 56, 728 (1939).

[53] A. Henstra, T.-S. Lin, J. Schmidt, and W. T. Wenckebach, High Dynamic Nuclear Polarization at Room Temperature, Chem. Phys. Lett. 165, 6 (1990).

[54] K. Tateishi, M. Negoro, S. Nishida, A. Kagawa, Y. Morita, and M. Kitagawa, Room Temperature Hyperpolarization of Nuclear Spins in Bulk, Proc. Natl. Acad. Sci. U.S.A. 111, 7527 (2014).

[55] S. Fujiwara, M. Hosoyamada, K. Tateishi, T. Uesaka, K. Ideta, N. Kimizuka, and N. Yanai, Dynamic Nuclear Polarization of Metal-Organic Frameworks Using Photoexcited Triplet Electrons, J. Am. Chem. Soc. 140, 15606 (2018).

[56] V. Jacques, P. Neumann, J. Beck, M. Markham, D. Twitchen, J. Meijer, F. Kaiser, G. Balasubramanian, F. Jelezko, and J. Wrachtrup, Dynamic Polarization of Single
Nuclear Spins by Optical Pumping of Nitrogen-Vacancy Color Centers in Diamond at Room Temperature, Phys. Rev. Lett. 102, 057403 (2009).

[57] A. L. Falk, P. V. Klimov, V. Ivády, K. Szász, D. J. Christle, W. F. Koehl, Á. Gali, and D.D. Awschalom, Optical Polarization of Nuclear Spins in Silicon Carbide, Phys. Rev. Lett. 114, 247603 (2015).

[58] J. P. King, K. Jeong, C. C. Vassiliou, C. S. Shin, R. H. Page, C. E. Avalos, H.-J. Wang, and A. Pines, Room-Temperature In Situ Nuclear Spin Hyperpolarization from Optically Pumped Nitrogen Vacancy Centres in Diamond, Nat. Commun. 6, 8965 (2015).

[59] G. Mathies, S. Jain, M. Reese, and R. G. Griffin, Pulsed Dynamic Nuclear Polarization with Trityl Radicals, J. Phys. Chem. Lett. 7, 111 (2016).

[60] W. J. Baker, K. Ambal, D. P. Waters, R. Baarda, H. Morishita, K. van Schooten, D. R. McCamey, J. M. Lupton, and C. Boehme, Robust Absolute Magnetometry with Organic Thin-Film Devices, Nat. Commun. 3, 898 (2012).

[61] J. Behrends, A. Sperlich, A. Schnegg, T. Biskup, C. Teutloff, K. Lips, V. Dyakonov, and R. Bittl, Direct Detection of Photoinduced Charge Transfer Complexes in Polymer Fullerene Blends, Phys. Rev. B 85, 125206 (2012).

[62] C. Boehme, D. R. McCamey, K. J. van Schooten, W. J. Baker, S.-Y. Lee, S.-Y. Paik, and J. M. Lupton, Pulsed Electrically Detected Magnetic Resonance in Organic Semiconductors, Phys. Status Solidi B 246, 2750 (2009).

[63] A. Schnegg, J. Behrends, M. Fehr, and K. Lips, Pulsed Electrically Detected Magnetic Resonance for Thin Film Silicon and Organic Solar Cells, Phys. Chem. Chem. Phys. 14, 14418 (2012).

[64] H. Malissa, M. Kavand, D. P. Waters, K. J. Van Schooten, P. L. Burn, Z. V. Vardeny, B. Saam, J. M. Lupton, and C. Boehme, Room-Temperature Coupling between Electrical Current and Nuclear Spins in OLEDs, Science 345, 1487 (2014).

[65] E. F. Thenell, M. E. Limes, E. G. Sorte, Z. V. Vardeny, and B. Saam, Nuclear Relaxation Measurements in Organic Semiconducting Polymers for Application to Organic Spintronics, Phys. Rev. B 91, 045205 (2015).

[66] Z. V. Vardeny, Organic Spintronics (CRC Press, Boca Raton, FL, 2010). 